\title{
Direct regulation of complex I by mitochondrial MEF2D is disrupted in a mouse model of Parkinson disease and in human patients
}

\author{
Hua She, ${ }^{1}$ Qian Yang, ${ }^{1}$ Kennie Shepherd, ${ }^{2}$ Yoland Smith,,3 Gary Miller,, ${ }^{1,2,3}$ \\ Claudia Testa, ${ }^{3}$ and Zixu Mao ${ }^{1,3}$ \\ ${ }^{1}$ Department of Pharmacology, ${ }^{2}$ Department of Environmental and Occupational Health, Rollins School of Public Health, \\ ${ }^{3}$ Department of Neurology, School of Medicine, and ${ }^{4}$ Yerkes National Primate Research Center, Emory University, Atlanta, Georgia, USA.
}

\begin{abstract}
The transcription factors in the myocyte enhancer factor 2 (MEF2) family play important roles in cell survival by regulating nuclear gene expression. Here, we report that MEF2D is present in rodent neuronal mitochondria, where it can regulate the expression of a gene encoded within mitochondrial DNA (mtDNA). Immunocytochemical, immunoelectron microscopic, and biochemical analyses of rodent neuronal cells showed that a portion of MEF2D was targeted to mitochondria via an $\mathrm{N}$-terminal motif and the chaperone protein mitochondrial heat shock protein 70 ( $\mathrm{mtHsp70}$ ). MEF2D bound to a MEF2 consensus site in the region of the mtDNA that contained the gene NADH dehydrogenase 6 (ND6), which encodes an essential component of the complex I enzyme of the oxidative phosphorylation system; MEF2D binding induced ND6 transcription. Blocking MEF2D function specifically in mitochondria decreased complex I activity, increased cellular $\mathrm{H}_{2} \mathrm{O}_{2}$ level, reduced ATP production, and sensitized neurons to stress-induced death. Toxins known to affect complex I preferentially disrupted MEF2D function in a mouse model of Parkinson disease (PD). In addition, mitochondrial MEF2D and ND6 levels were decreased in postmortem brain samples of patients with PD compared with age-matched controls. Thus, direct regulation of complex I by mitochondrial MEF2D underlies its neuroprotective effects, and dysregulation of this pathway may contribute to PD.
\end{abstract}

\section{Introduction}

Mitochondria are the primary energy-generating organelles in most eukaryotic cells. In addition, they also participate in metabolism, calcium signaling, and apoptosis (1, 2). Mitochondrial dysfunction and the ensuing oxidative stress cause damages to key cellular macromolecules, including DNA, which affects basic biological processes ranging from bioenergetics to gene transcription to structural integrity. These detrimental effects have been proposed to play important roles in aging, metabolic disorders, and particularly neurodegeneration $(3,4)$. Strong evidence from molecular studies, genetic analyses, and mouse models shows that etiological factors associated with Alzheimer disease, Parkinson disease (PD), Huntington disease, amyotrophic lateral sclerosis, hereditary spastic paraplegia, and cerebellar degenerations lead to mitochondrial impairment and may contribute to the pathogenesis of these disorders (5). Therapeutic approaches targeting mitochondrial dysfunction have great promise but require a much better understanding of fundamental mitochondrial biology and how it may be dysregulated under pathological conditions.

Mitochondrial DNA (mtDNA) encodes some of the components of enzymatic complexes for oxidative phosphorylation. Proper assembly of a functional oxidative phosphorylation system requires coordination of mitochondrial and nuclear gene expression. The individual strands of the supercoiled circular mtDNA are denoted heavy $(\mathrm{H})$ strand and light $(\mathrm{L})$ strand based on their

Conflict of interest: The authors have declared that no conflict of interest exists. Citation for this article: J Clin Invest. 2011;121(3):930-940. doi:10.1172/JCI43871. different buoyant densities. Of the 13 proteins determined by mtDNA, only 1 polypeptide - NADH dehydrogenase 6 (ND6), an essential component of complex I (6) - is encoded by the L strand (7). Mutations in the ND6 gene or alteration in its protein level have been linked etiologically to Leber hereditary optic neuropathy (LHON) (8) and associated with $\operatorname{PD}(9,10)$. The basic mtDNA transcription machinery consists of 1 mitochondrial RNA polymerase and 3 transcription factors: mitochondrial transcription factor A (TFAM), mitochondrial transcription factor B1 (TFB1M), and mitochondrial transcription factor B2 (TFB2M), which bind to the D-loop promoters in mtDNA to stimulate transcription. However, whether there are mechanisms that control strand-specific transcription of mtDNA and how they may be dysregulated under pathological stress remain unknown (11).

Various isoforms of transcription factor MEF2 (MEF2A-MEF2D) constitute a group of nuclear proteins found to play important roles in increasing types of cells. For example, MEF2s have been shown to regulate immune cell response, control glucose metabolism in adipocytes, participate in angiogenesis, promote liver fibrosis, and modulate muscle cell differentiation. Many key signaling mechanisms converge on MEF2 to regulate its activity (12). In neurons, MEF2s are required to regulate neuronal development, synaptic plasticity, and survival (13). Indeed, MEF2s promote the survival of several types of neurons under different conditions (14-17). In cellular models, negative regulation of MEF2s by stress and toxic signals contributes to neuronal death (18). In contrast, enhancing MEF2 activity not only protects primary neurons from death, but also attenuates the loss of dopaminergic (DA) neurons in substantia nigra 
A

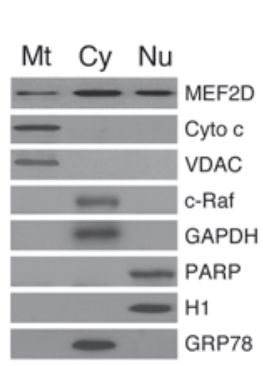

B
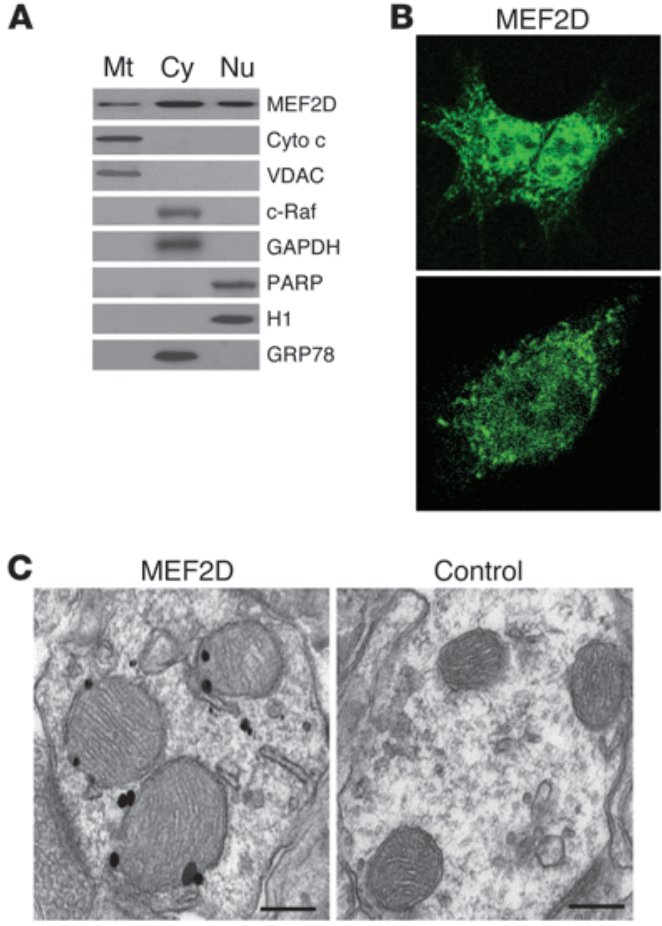

Control

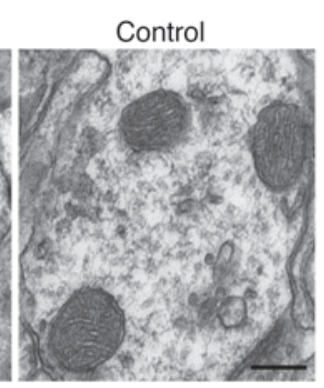

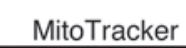
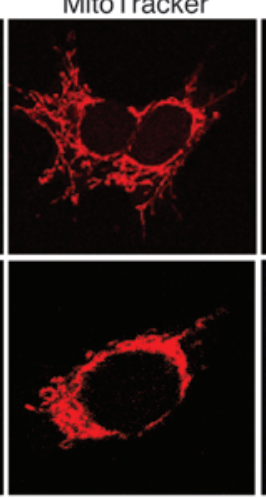

D

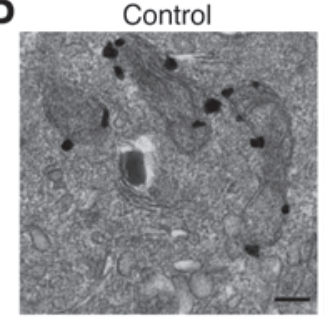

$\mathbf{F}$

DAPI
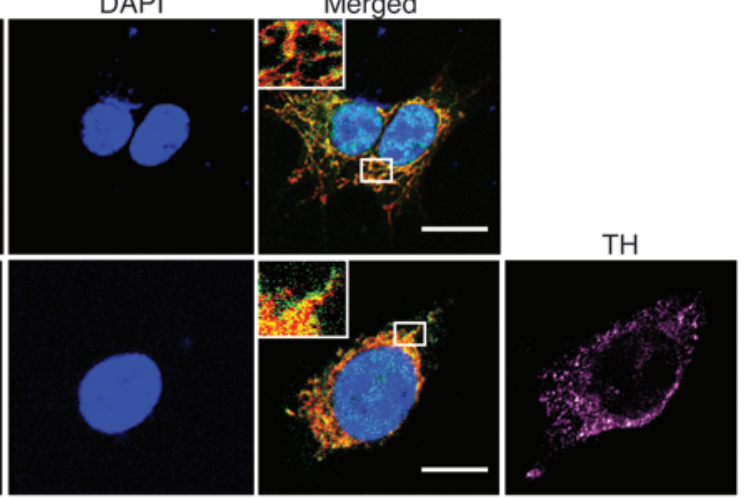

SiRNA

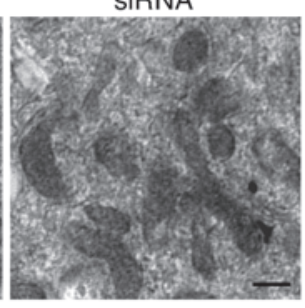

E Om IMS Im Ma
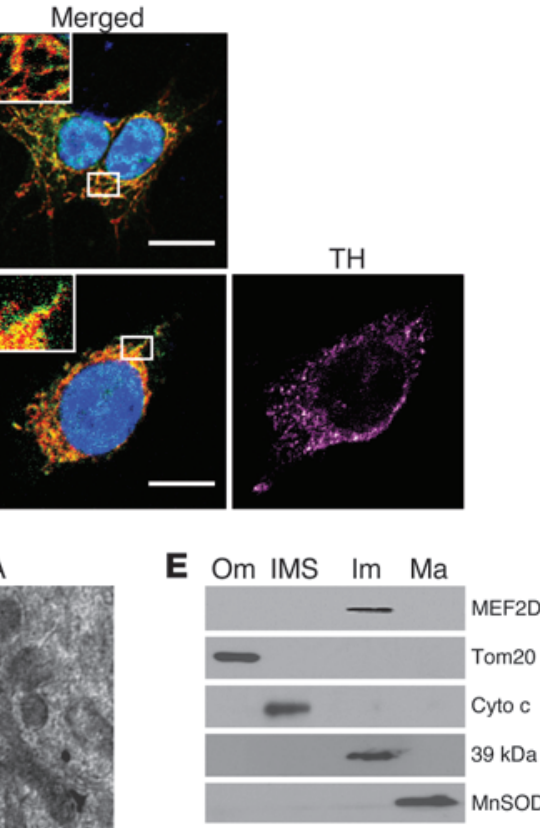

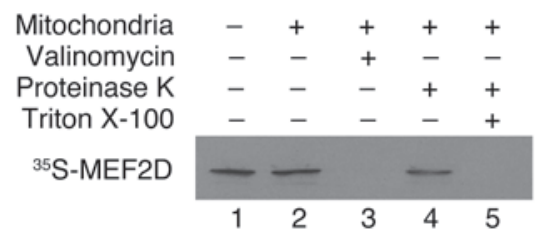

\section{Figure 1}

Localization of MEF2D in mitochondria of neuronal cells. (A) Localization of MEF2D in mitochondria of SN4741 cells $(n=3)$. Cyto c and VDAC are mitochondrial (Mt) markers; c-Raf and GAPDH are cytoplasmic (Cy) markers; PARP and histone H1 are nuclear (Nu) markers; GRP78 is a ER marker. (B) Colocalization of MEF2D with MitoTracker in both SN4741 cells and primary rat midbrain DA neurons $(n=4)$. TH is a DA neuron marker. Scale bars: $10 \mu \mathrm{m}$. Inset original magnification, $\times 1,000$. (C) Localization of MEF2D in rat brain mitochondria under TEM. The control is without primary antibody $(n=4)$. Scale bars: $100 \mathrm{~nm}$. (D) Localization of MEF2D in mitochondria of SN4741 cells under TEM $(n=4)$. Scale bars: $50 \mathrm{~nm}$. (E) Localization of MEF2D in the inner membrane of rat brain mitochondria. Tom20, Cyto c, complex I 39-kDa protein, and MnSOD are markers for mitochondrial outer membrane (Om), inter-membrane space (IMS), inner membrane (Im), and matrix (Ma), respectively. $n=3$. (F) In vitro mitochondrial import of MEF2D. Lane 1, MEF2D control (1:10 input); lane 2, imported MEF2D; lane 3, valinomycin-induced (20 $\mu$ M) loss of membrane potential on MEF2D import; lane 4, resistance to proteinase K digestion after MEF2D import; lane 5, complete digestion of MEF2D by proteinase $\mathrm{K}$ after solubilization of mitochondria with Triton $\mathrm{X}-100 . n=3$.

pars compacta $(\mathrm{SNpc})$ in a 1-methyl 4-phenyl 1,2,3,6-tetrahydropyridine (MPTP) mouse model of PD (19). The precise mechanisms by which MEF2s promote neuronal survival are not fully understood, although it is assumed that MEF2s exert their effects, like in other cell systems, by directly regulating the expression of nuclear target genes, given their well-established presence in the nucleus (20). Here we report the unexpected finding of MEF2D in mitochondria and its role therein. We identified the mtDNA-encoded ND6 gene as the direct target regulated by MEF2D. Disruption of this pathway underlaid neurotoxicity induced by toxic signals relevant to PD in culture and animal models and was found in the postmortem brains of PD patients.

\section{Results}

Localization of MEF2D in mitochondria of neuronal cells. The finding that MEF2s are involved in mitochondrial biogenesis (21) prompted us to ask whether MEF2s directly regulate mito- chondrial function. To determine mitochondrial localization of MEF2D, we prepared purified mitochondrial fractions from SN4741 cells, a mouse DA neuronal cell line expressing tyrosine hydroxylase (TH) (Supplemental Figure 1; supplemental material available online with this article; doi:10.1172/JCI43871DS1) and widely used in the study of neuronal toxins (22). Western blot analysis showed that MEF2D was present in highly purified mitochondrial fractions (Figure 1A). The cytoplasmic (c-Raf and GAPDH) and nuclear (PARP and H1) markers were only detected in their respective fractions, which indicated that the mitochondrial preparations were not contaminated with other subcellular fractions. The monoclonal antibody used to probe MEF2D was very specific: it recognized a single band on Western blot that was reduced to background by MEF2D siRNA (Supplemental Figure 2). Consistent with our Western blot findings, immunocytochemical studies confirmed colocalization of MEF2D with the mitochondria-specific fluorescent dye 
A
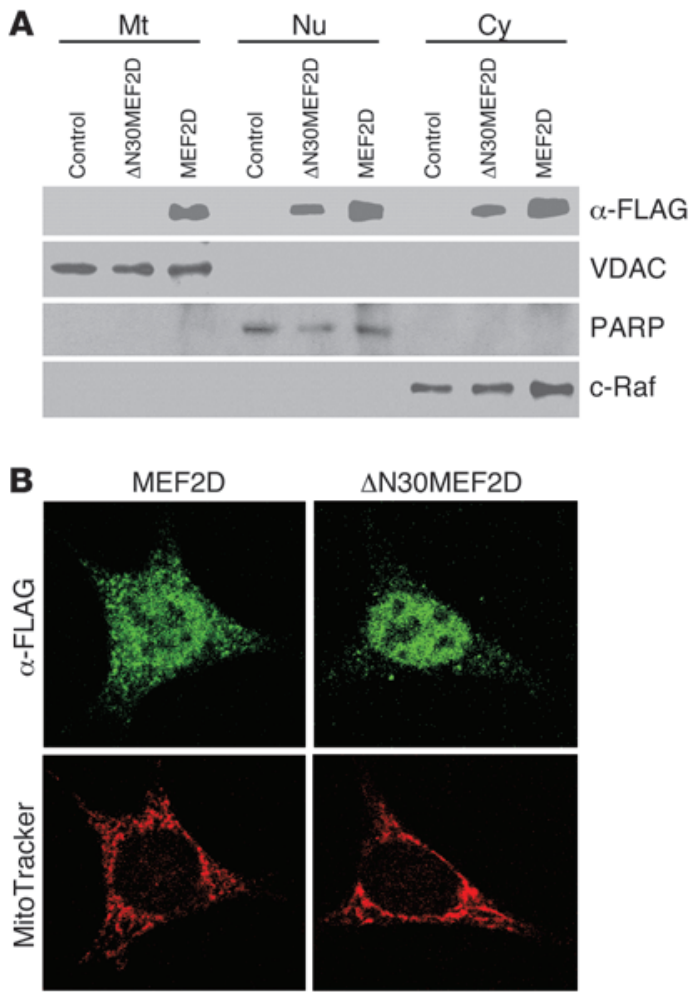

$\triangle \mathrm{N} 30 \mathrm{MEF} 2 \mathrm{D}$
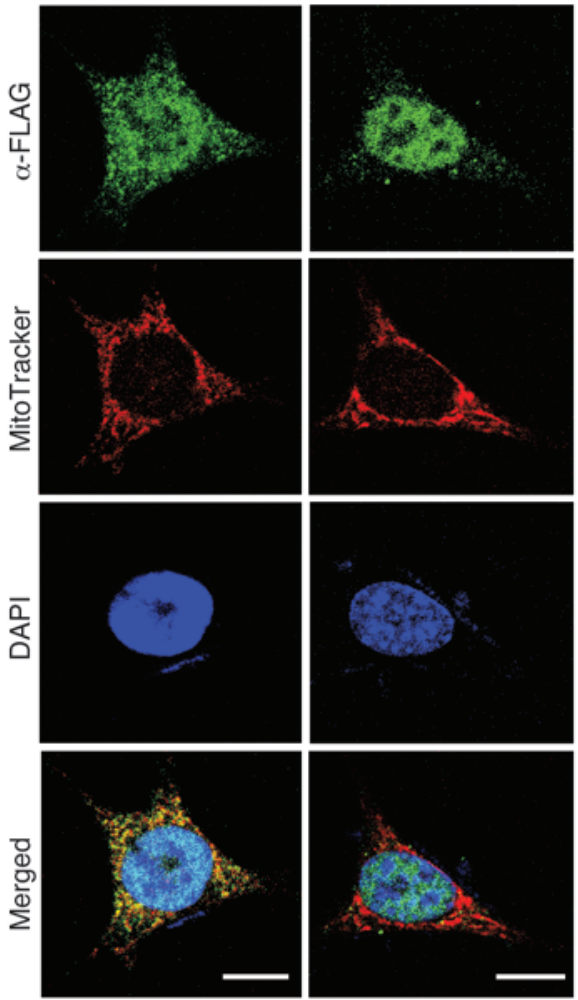

C

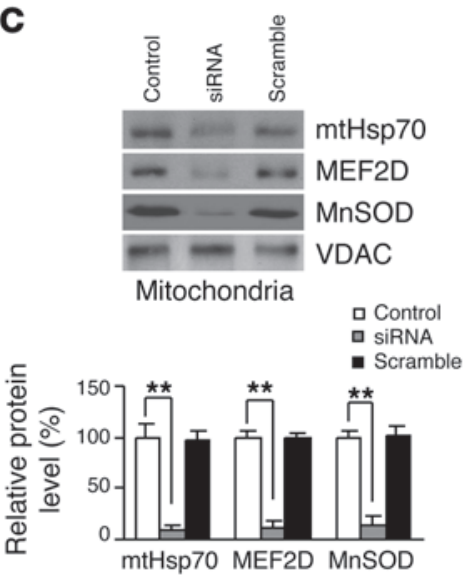

D
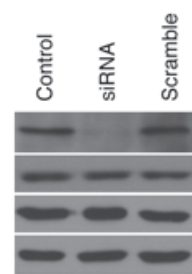

$\mathrm{mtHsp} 70$

MEF2D

MnSOD

Actin

Whole cell

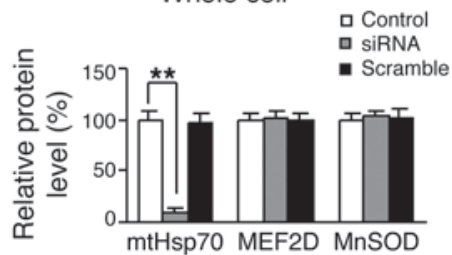

\section{Figure 2}

Specific sequence and chaperone protein required for localization of MEF2D to mitochondria. (A) Lack of mitochondrial localization by $\triangle$ N30MEF2D. Western blotting showed the presence of overexpressed $\triangle N 30 M E F 2 D$ in cytoplasmic and nuclear fractions, but not in the mitochondrial fraction, of SN4741 cells $(n=3)$. VDAC, PARP, and c-Raf are mitochondrial, nuclear, and cytoplasmic markers, respectively. Control indicates the control vector group. (B) Immunocytochemistry analysis of mitochondrial localization of transfected MEF2D-Flag. Overexpressed $\triangle$ N30MEF2D did not colocalize with MitoTracker in SN4741 cells $(n=50$ cells; $\left.{ }^{* \star} P<0.01\right)$. Experiments were repeated 4 times. Scale bars: $15 \mu \mathrm{m}$. (C and D) Requirement of mtHsp70 for mitochondrial targeting of MEF2D $(n=4$; $\left.{ }^{\star \star} P<0.01\right)$. Control indicates untreated. Knocking down mouse mtHsp70 by siRNA reduced MEF2D level in purified mitochondria from SN4741 cells (C). Knocking down mouse mtHsp70 by siRNA did not reduce whole cell MEF2D level in SN4741 cells (D). MnSOD is a known mtHsp70-imported mitochondrial matrix protein.
MitoTracker in cultured SN4741 cells and primary midbrain DA neurons (Figure 1B and Supplemental Figure 3). MEF2D siRNA reduced the MEF2D-MitoTracker colocalization signal (Supplemental Figure 3). We further investigated the ultrastructural distribution of MEF2D by immunogold transmission electron microscopy (TEM). Specific immunogold particles were preferentially found in mitochondria of rat brain and SN4741 cells. This immunogold particle distribution was not observed when the primary anti-MEF2D antibody was omitted and was greatly reduced by MEF2D siRNA (Figure 1, C and D). To localize MEF2D within mitochondria biochemically, we performed subfractionation of highly purified rat brain mitochondria. Western blot analysis of the subfractions showed that MEF2D was present predominately in the inner membrane of mitochondria (Figure 1E). In vitro mitochondrial import assay showed that MEF2D translated in vitro was imported into isolated energized mitochondria (Figure 1F). Collectively, these data demonstrated that a portion of MEF2D in neurons is localized to mitochondria and enriched in the mitochondrial inner membrane.
Specific sequence and chaperone protein required for localization of $M E F 2 D$ to mitochondria. Proteins are often targeted to mitochondria if their sequence contains a mitochondria-targeting motif; sometimes, they require the aid of chaperones (23). The structure of MEF2D is divided into the smaller N-terminal DNA-binding domain and the larger C-terminal transactivation domain (20). The N-terminal 30 aa residues of MEF2D are involved in protein interactions including chaperones and are predicted to have a weak motif for mitochondrial localization signal by iPSORT (http://hc.ims.u-tokyo.ac.jp/iPSORT/). To verify this prediction, we generated a MEF2D-Flag mutant with the putative mitochondrial targeting signal deleted $(\triangle N 30 M E F 2 D)$ and studied its subcellular distribution (Supplemental Figure 4). When overexpressed in SN4741 cells, WT MEF2D-Flag was detectable in nuclear, cytoplasmic, and mitochondrial fractions (Figure 2A), just as the endogenous MEF2D was (Figure 1A). The $\triangle$ N30MEF2D-Flag mutant, when transfected into cells, was well expressed and readily detectable in whole cell lysate. However, unlike its WT counterpart, $\triangle$ N30MEF2D-Flag mutant was 


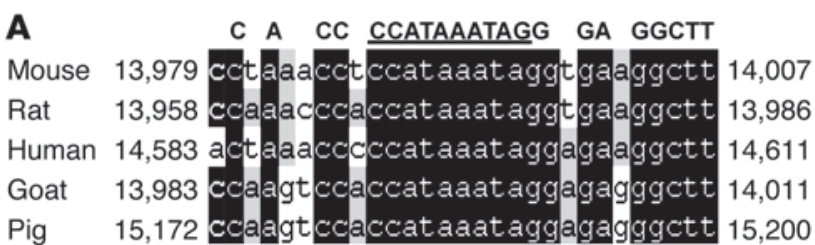

\begin{tabular}{|c|c|c|c|c|}
\hline GST & - & + & - & - \\
\hline Mut probe (hot) & - & - & - & - \\
\hline WT probe (hot) & + & + & + & 1 \\
\hline WT probe (cold) & - & - & - & 100 \\
\hline ST-MEF2D (1-91) & - & - & + & + \\
\hline
\end{tabular}

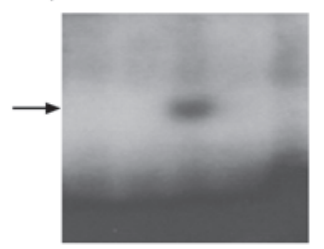

D
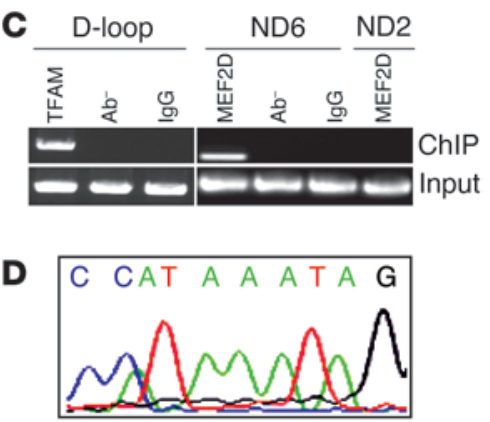

Figure 3

Identification of MEF2D regulatory target in mtDNA. (A) Presence of a conserved MEF2 consensus site in ND6 gene in mtDNA of different species. Underlined sequence indicates the MEF2 consensus site. Black-shaded areas show the conversed MEF2 site and sequences around it in ND6. (B) Specific binding of MEF2D to the consensus site in ND6 in vitro $(n=3)$. EMSA assay revealed that MEF2D bound to WT but not mutant (Mut) probe. Arrow indicates the specific binding complex. GST, glutathione S-transferase; GST-MEF2D(1-91), GST-fused MEF2D1-91 aa. Hot and cold refer to labeled and unlabeled probes, respectively. (C) Binding of MEF2D to the consensus site in ND6 in cells in vivo $(n=3)$. ChIP assay showed that MEF2D binds to ND6 in SN4741 cells. A fragment bound by anti-MEF2D antibody could only be specifically amplified by PCR with ND6 primers after immunoprecipitation. TFAM, a known mtDNA D-loop binding protein, was used as a control. $\mathrm{Ab}^{-}$, without primary antibody. (D) Sequence analysis of the purified PCR fragment bound by anti-MEF2D antibody in $\mathbf{C}$ confirmed that it is the predicted ND6 fragment and contains the MEF2 consensus sequence.

absent from the mitochondrial fraction, but detectable in the cytoplasmic and nuclear fractions (Figure 2A). Consistent with this, immunocytochemical studies showed that, in contrast to WT MEF2D-Flag, $\triangle$ N30MEF2D-Flag largely lost its colocalization with MitoTracker in SN4741 cells (Figure 2B).

Mitochondrial heat shock protein 70 ( $\mathrm{mtHsp} 70)$ is a key component of the mitochondrial import machinery $(23,24)$. We tested whether blocking $\mathrm{mtHsp} 70$ affects localization of MEF2D to mitochondria by transfecting SN4741 cells with siRNA oligos targeting mouse mtHsp70 and then examining MEF2D in purified mitochondria. Mouse mtHsp70 siRNA led to a marked reduction of $\mathrm{mtHsp} 70$ protein level. This was accompanied by a similar decline in the levels of mitochondrial MEF2D and manganese superoxide dismutase (MnSOD) (Figure $2 \mathrm{C}$ ), the latter of which is known to require $\mathrm{mtHsp} 70$ for its mitochondrial localization. On the other hand, the level of MEF2D in whole cell lysate was not affected (Figure 2D), which indicates that translocation of MEF2D into mitochondria is specifically regulated by $\mathrm{mtHsp} 70$.

Identification of $M E F 2 D$ regulatory target in $m t D N A$. The specific targeting of MEF2D to mitochondria prompted us to search for possible MEF2 regulatory targets in the mtDNA. Analyzing the entire mtDNA revealed the presence of a single putative MEF2 consensus site $\left(5^{\prime}-\mathrm{CC}[\mathrm{A} / \mathrm{t}][\mathrm{t} / \mathrm{a}] \mathrm{AAATAG}-3^{\prime}\right)(25)$ in the coding region of the ND6 gene (Figure $3 \mathrm{~A})$. Moreover, this putative MEF2 binding site was conserved among several species. To test whether
MEF2D binds to this putative MEF2 site, we performed EMSA. N-terminal MEF2D formed a complex with the labeled probe containing the ND6 MEF2 site. Mutation of the ND6 MEF2 site disrupted the complex formation (Figure 3B), which suggests that MEF2D specifically binds to this site in vitro. To corroborate this finding, we carried out ChIP assay using highly purified mitochondria. Our ChIP analysis showed that MEF2D bound specifically to this ND6 region in SN4741 cells, as ND6- but not ND2-based primers amplified the bound sequence (Figure 3C). Sequence analysis of the DNA bound by MEF2D after amplification confirmed the presence of the ND6 MEF2 site (Figure 3D).

Transcriptional regulation of mitochondrial gene $N D 6$ by mitochondrial MEF2D. To assess the function of MEF2D specifically in mitochondria without affecting nuclear MEF2, we generated MEF2D mutants (active and dominant-negative MEF2D; referred to herein as Mt2D and Mt2Ddn, respectively) that lack the nuclear localization signals at the very $\mathrm{C}$ terminus of the protein and carry an additional mitochondriatargeting signal fused to the $\mathrm{N}$ terminus (Figure 4A). These mutants displayed an enhanced ability to preferentially localize to the mitochondria versus the nucleus. When overexpressed in SN4741 cells, they colocalized with MitoTracker in the cytoplasm with greatly reduced nuclear presence, as analyzed by immunocytochemistry (Supplemental Figure 5A). MEF2 reporter assay, which measures nuclear MEF2 activity, showed that, unlike their nuclear counterparts, neither Mt2D nor Mt2Ddn affected the expression of nuclear MEF2 reporter (Supplemental Figure 5B). When overexpressed in SN4741 cells, both Mt2D and Mt2Ddn modulated the binding of full-length MEF2D to the ND6 MEF2 site, as shown by ChIP assay (Figure 4B). Interestingly, although it bound to the ND6 MEF2 site at a high level, Mt2D did not significantly change the level of ND6 protein, whereas Mt2Ddn caused a marked reduction (Figure 4C). Furthermore, Mt2Ddn did not alter the levels of PGC1, a known nuclear target of MEF2, which suggests that Mt2Ddn preferentially changes the MEF2D target in mitochondria but not in the nucleus. ND6 is a key component for oxidative phosphorylation complex I. To assess whether the effect of Mt2Ddn on ND6 is specific, we determined the mRNA levels for the components of various other oxidative phosphorylation complexes. Mt2Ddn had little effect on the mRNA levels of other mitochondria-encoded proteins tested under the condition in which it clearly reduced ND6 mRNA (Figure 4D). To further confirm that mitochondrial MEF2D is required for ND6 expression, we knocked down MEF2D by siRNA in SN4741 cells and found that ND6 level was indeed reduced (Supplemental Figure 6C). An active MEF2D targeted to mitochondria and not affected by MEF2D siRNA (Mt2DVP16) completely blocked MEF2D siRNA-induced reduction of ND6 level (Supplemental Figure 6C). To explore how MEF2D regulates ND6 gene expression, we performed in vitro mtDNA L-strand transcription assay. Our data showed that Mt2D increased, whereas Mt2Ddn reduced, the level of L-strand promoter transcripts (Figure 4E). Consistent 
A

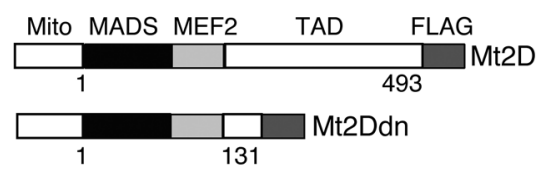

D

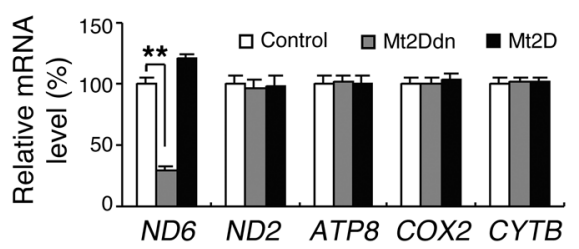

B

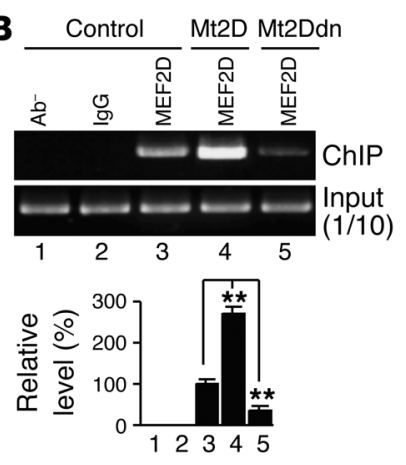

E

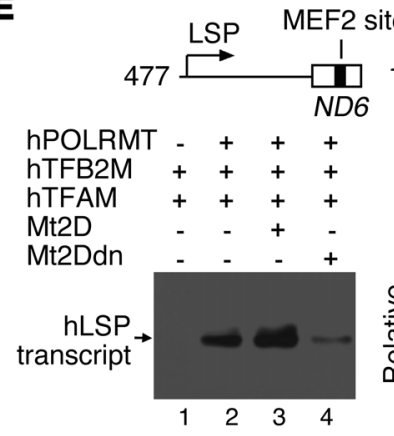

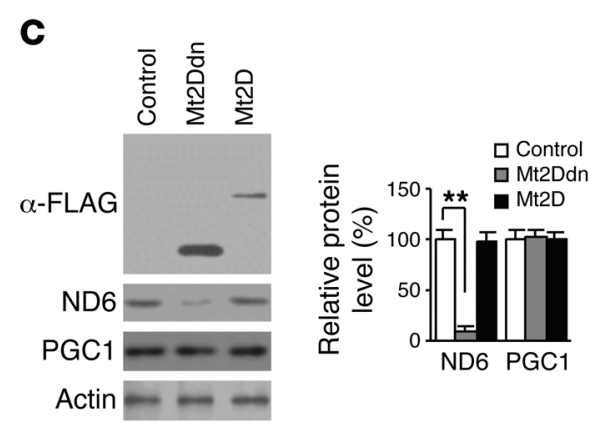
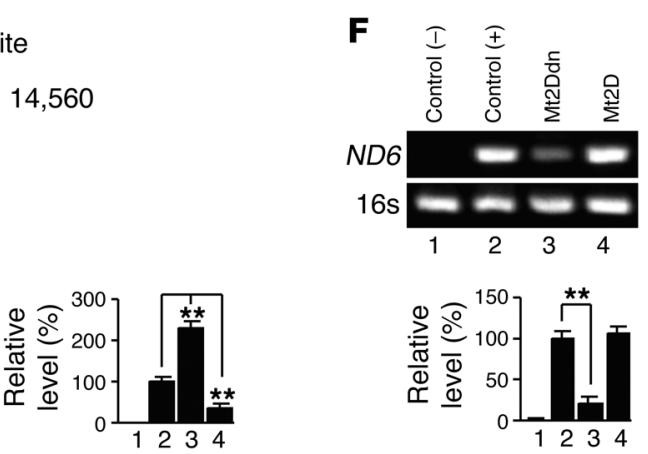

\section{1.s.so}<smiles>CCC</smiles>

\section{Figure 4}

Regulation of mitochondrial gene ND6 by mitochondrial MEF2D. (A) Mt2D and Mt2Ddn, which lacks the transactivation domain (TAD). Mito, mitochondrial targeting sequence; MADS, minichromosome maintenance 1, agamous, deficiens, and serum response factor domain. (B) Effects of Mt2D and Mt2Ddn on binding of full-length MEF2D to ND6 gene in mitochondria of SN4741 cells, revealed by ChIP assay $\left(n=4\right.$; $\left.{ }^{* \star} P<0.01\right)$. Control indicates the control vector group. (C) Effects of Mt2D or Mt2Ddn on ND6 expression in SN4741 cells. Overexpression of Mt2Ddn in SN4741 cells reduced ND6, but not PPAR- $\gamma$ coactivator 1 (PGC1), expression $\left(n=4 ;{ }^{* \star} P<0.01\right.$ ). Control indicates the control vector group. (D) Effects of overexpression of Mt2D or Mt2Ddn on mRNA levels of mitochondria encoded genes. Real-time PCR results showed specific reduction of ND6 mRNA level by overexpression of Mt2Ddn in SN4741 cells $\left(n=4 ;{ }^{* *} P<0.01\right)$. Control indicates the control vector group. (E) Effects of Mt2D or Mt2Ddn on mtDNA L-strand transcription initiation in vitro $\left(n=3 ;{ }^{* *} P<0.01\right)$. A human mtDNA fragment containing mitochondrial L-strand promoter (LSP) and ND6 gene was used in the in vitro transcription assay. Blot shows ${ }^{32} \mathrm{P}-\mathrm{UTP}-$ labeled transcripts. (F) Effects of Mt2D or Mt2Ddn on mtDNA de novo transcription in vivo $\left(n=3 ;{ }^{* *} P<0.01\right)$. Control(-) is without primers. Control(+) indicates the control vector group.

with these data, Mt2Ddn reduced the de novo transcription of ND6 in vivo (Figure 4F). These findings suggest that mitochondrial MEF2D activity is specifically required for the transcription of mitochondrial ND6 gene driven by L-strand promoter.

Specific modulation of complex I activity by mitochondrial MEF2D. ND6 has previously been reported to be required for the proper assembly of complex I (6). We tested whether activity of mitochondrial MEF2D affects complex I function. Overexpression of Mt2Ddn in SN4741 cells markedly reduced the protein level of mitochondrial complex I, but had no effect on complexes II-V, by nondenatured gel electrophoresis (Figure 5A). Similarly, Mt2Ddn also significantly reduced mitochondrial complex I activity, but not that of complexes III-V (Figure 5, A and $\mathrm{C}$ ). Interestingly, $\mathrm{Mt} 2 \mathrm{D}$ increased neither the protein level nor the activity of complex I (Figure 5, A and C). To further demonstrate that mitochondrial MEF2D-ND6 is required for maintaining complex I activity, we overexpressed ND6 via a construct (Supplemental Figure 6, A and B) that was not regulated by mitochondrial MEF2D; this rescued complex I activity from Mt2Ddn-induced inhibition (Figure 5B). Together, these results demonstrate that the activity of mitochondrial MEF2D is specifically required for complex I function. To corroborate the role of MEF2D in the regulation of complex I, we determined whether
MEF2D affects mitochondrial function. Mt2Ddn led to a clear reduction in cellular ATP level and significantly increased $\mathrm{H}_{2} \mathrm{O}_{2}$ production by mitochondria; however, it did not significantly alter mitochondrial membrane potential (Figure 5D). Consistent with findings in Figure 5, A and B, Mt2D did not alter the levels of ATP, $\mathrm{H}_{2} \mathrm{O}_{2}$, and mitochondrial membrane potential. These data indicate that direct regulation of ND6 by MEF2D is critical and required for proper mitochondrial function.

Inhibition of mitochondrial MEF2D by toxic signals relevant to $P D$. Mitochondrial dysfunction and oxidative stress have been implicated in the pathogenesis of PD (26). This led us to test whether toxic signals implicated in $\mathrm{PD}(27,28)$ regulate mitochondrial MEF2D. We treated SN4741 cells with the toxicants 1-methyl4-phenylpyridinium (MPP+) and rotenone and performed ChIP assays. Relatively short exposure to these toxicants greatly reduced the binding of mitochondrial MEF2D to the ND6 MEF2 site and the mRNA level of ND6 (Figure 6A and Supplemental Figure 7A). Consistently, exposure to $\mathrm{MPP}+$ and rotenone also led to a decrease in the levels of mitochondrial MEF2D and ND6 proteins, whereas under the same condition, the levels of MEF2D in the nucleus and in whole cells were not affected (Figure 6B and Supplemental Figure 7B). To support these Western blot findings, we performed immunocytochemistry studies in SN4741 
A
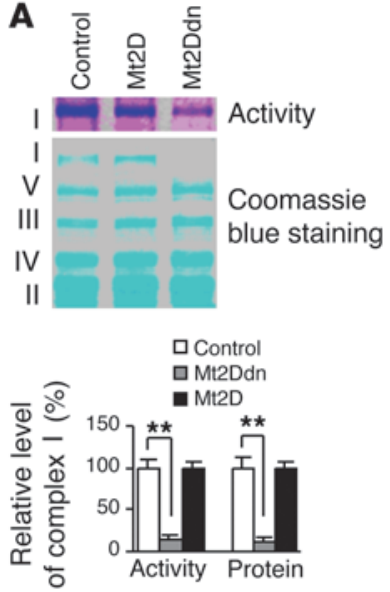
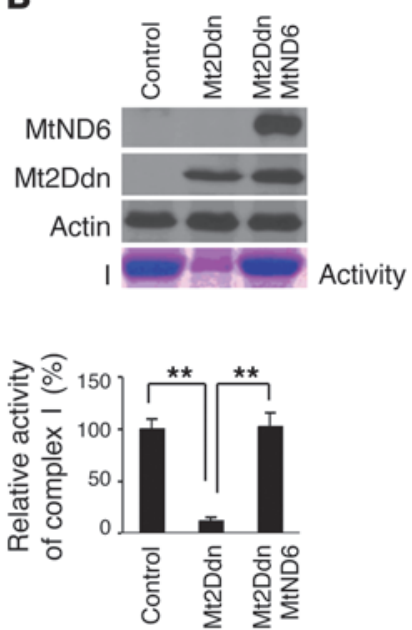

C

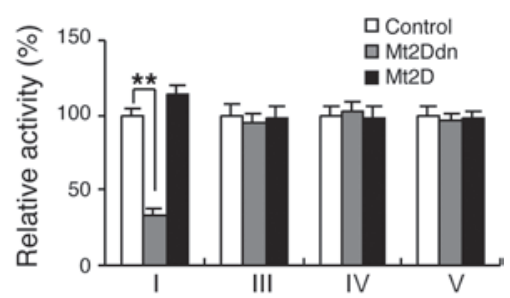

D

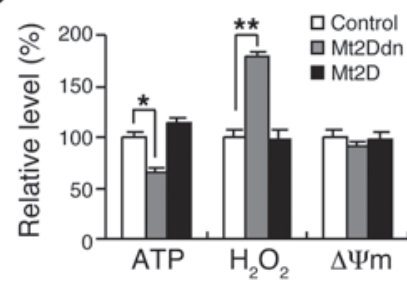

Figure 5

Specific modulation of complex I activity by mitochondrial MEF2D. (A) Requirement of mitochondrial MEF2D for complex I activity. Overexpression of Mt2Ddn reduced mitochondrial complex I activity, revealed by BN-PAGE and in-gel activity staining. Coomassie blue staining showed specific reduction of complex I protein level after overexpression of Mt2Ddn $\left(n=3 ;{ }^{*} P<0.01\right)$. Control indicates the control vector group. (B) Overexpression of ND6 rescued complex I activity reduced by Mt2Ddn. Control indicates the control vector group. (C) Measurement of various complex activities. Quantitative analysis of mitochondrial complex activities showed specific reduction of complex I activity by Mt2Ddn in SN4741 cells $\left(n=4 ;{ }^{* *} P<0.01\right)$. Control indicates the control vector group. (D) Effect of overexpression of Mt2D or Mt2Ddn on mitochondrial function. Mt2Ddn reduced cellular ATP level and elevated $\mathrm{H}_{2} \mathrm{O}_{2}$ production in SN4741 cells $\left(n=4 ;{ }^{*} P<0.05\right.$, $\left.{ }^{* \star} P<0.01\right)$. $\Delta \psi \mathrm{m}$, loss of mitochondrial membrane potential. Control indicates the control vector group.

cells and in cultured primary midbrain DA neurons. MPP+ and rotenone preferentially reduced colocalization of MEF2D and MitoTracker signals in these neuronal cells (Figure 6C and Supplemental Figure 8).

To assess the role of mitochondrial MEF2D in neuronal survival, we exposed SN4741 cells to different doses of MPP+ known to cause selective degeneration of DA neurons in vivo and measured cellular viability by 4-[3-(4-lodophenyl)-2-(4-nitrophenyl)-2H-5tetrazolio]-1,3-benzene disulfonate (WST-1) assay. MPP+ caused loss of neuronal viability in a dose-dependent manner (Figure 6D). Blocking mitochondrial MEF2D with Mt2Ddn clearly sensitized the cells to low-dose MPP+ toxicity, as measured by WST-1 assay, and Mt2Ddn also impaired cellular viability over time (Figure 6D). Consistent with these results, immunocytochemical studies revealed that Mt2Ddn increased the number of propidium iodide-positive cells following MPP+ treatment (Supplemental Figure 9). In agreement with the findings that constitutively active Mt2DVP16 enhanced ND6 expression (Supplemental Figure 6) and that MtND6 overexpression restored complex I activity (Figure 5B), overexpression of Mt2DVP16 or MtND6 in mitochondria partially attenuated MPP+-induced neuronal death (Figure 6D).

Correlation of mitochondrial MEF2D in MPTP model of PD and in postmortem brains of PD patients. Mice treated with the neurotoxin MPTP exhibit classic pathological and behavioral features observed in Parkinsonism and are widely used to model the disease (27). We examined the effects of this toxin on mitochondrial MEF2D in vivo using the brains of MPTP-treated mice. Chronic MPTP exposure caused a loss of immunohistochemical signal for TH in SNpc and striatum (Supplemental Figure 10A). This correlated well with a significant reduction of mitochondrial MEF2D in MPTP-treated mouse brain compared with saline-treated controls (Supplemental Figure 8, A and B). Furthermore, MPTP also led to a clear decline in the level of ND6 protein (Figure 7A). To show that inhibition of mitochondrial MEF2D plays a direct role in the loss of DA neurons in animal model of $\mathrm{PD}$, we injected recombinant lentivirus encoding Mt2Ddn, Mt2DVP16, or MtND6 into the SNpc of mouse brain and then exposed mice to MPTP. Quantification of TH showed that loss of MEF2D function in mitochondria significantly accelerated the loss of TH signal in DA neurons in vivo (Figure 7B). Overexpression of Mt2DVP16 or MtND6 in mitochondria partially attenuated MPTP-induced neuronal death (Figure 7B). To probe the relevance of mitochondrial MEF2D to human disease, we assessed the levels of mitochondrial MEF2D and ND6 proteins in postmortem brain samples of PD patients. Immunoblotting analysis revealed that the level of mitochondrial MEF2D was preferentially reduced in PD patients compared with matched controls (Figure 7C), which correlates closely with a significant reduction in the level of ND6 protein.

\section{Discussion}

Nuclear transcription factor MEF2s are involved in a growing number of critical cellular functions involving both neuronal and non-neuronal systems. The basic assumption has always been that MEF2s exert their control on cells solely through modulating the expression of nuclear target genes (20). Indeed, MEF2A has previously been shown to affect mitochondrial function by regulating the expression of mitochondrial proteins encoded by the nuclear genes (21). In this study, we provide the first evidence to our knowledge showing that MEF2D is present in neuronal mitochondria, where it binds to a discrete and well-conserved MEF2 consensus site within the coding region of mitochondrial gene ND6 to regulate its transcription, thereby directly modulating complex I activity and affecting a number of key mitochondrial functions and physiology. Thus, we believe MEF2D to qualify as a bona fide, novel mitochondrial transcrip- 
A

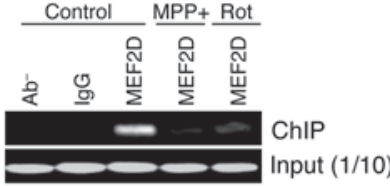

C
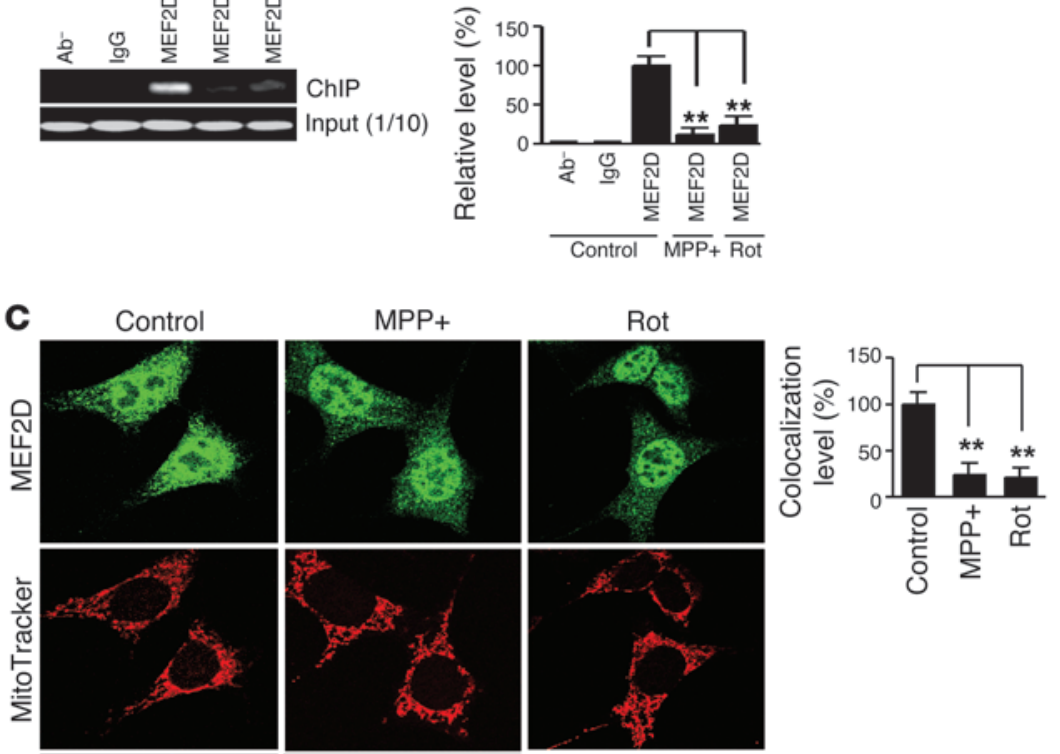

B
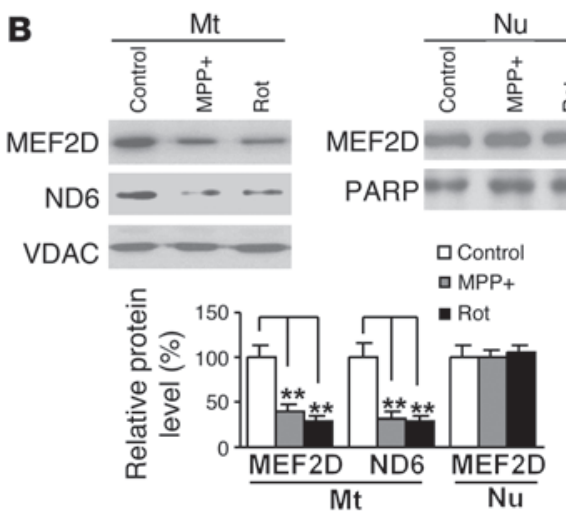

D
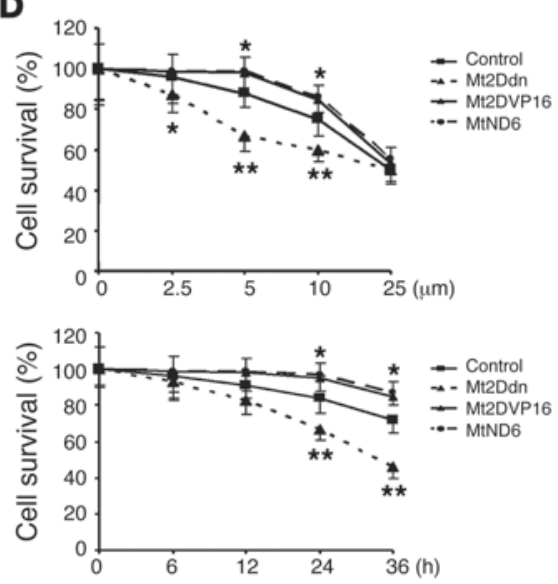

Figure 6

Inhibition of mitochondrial MEF2D by toxic signals relevant to PD. (A) Reduced binding of MEF2D to ND6 after neurotoxin treatment. SN4741 cells were treated with MPP $+(25 \mu \mathrm{M})$ or rotenone (Rot, $100 \mathrm{nM})$ for 12 hours. ChIP assay showed that binding of MEF2D to ND6 was greatly reduced $\left(n=4 ;{ }^{\star \star} P<0.01\right)$. Control indicates untreated. (B) Reduced mitochondrial MEF2D and ND6 protein levels after neurotoxin treatment. Western blotting showed that levels of MEF2D and ND6 in purified mitochondria, but not in nuclei, were significantly reduced $\left(n=4\right.$; $\left.{ }^{* \star} P<0.01\right)$. Control indicates untreated. (C) Immunocytochemical analysis of mitochondrial MEF2D after MPP+ and rotenone treatment. MPP+ and rotenone preferentially reduced colocalization of MEF2D with MitoTracker $\left(n=50\right.$ cells; $\left.{ }^{\star \star} P<0.01\right)$. Scale bars: $10 \mu \mathrm{m}$. Experiments were repeated 4 times. Control indicates untreated. (D) Effect of mitochondrial MEF2D-ND6 pathway on MPP+ toxicity in SN4741 cells. SN4741 cells were treated with MPP+ after infection with the control or with Mt2Ddn, Mt2DVP16, or MtND6 lentiviruses. Treatment was either with different doses for 24 hours (top) or the $5-\mu \mathrm{M}$ dose for different times (bottom). Cell viability was measured by WST-1 assay $\left(n=4 ;{ }^{*} P<0.05 ;{ }^{* *} P<0.01\right)$. Control indicates the control vector group.

tion factor. Our findings broaden the cellular roles played by MEF2D and raise a series of interesting questions, such as how nuclear and mitochondrial MEF2 activities are coordinated; what signal pathways are involved specifically in modulating mitochondrial MEF2D; whether unique posttranslational modifications are required for regulation of MEF2D in mitochondria; and what interactions may exist between mitochondrial MEF2D and other components of mitochondrial transcriptional machinery. Our recent study showed that the autophagic pathway regulates the activity of nuclear MEF2D (29); thus, it would be interesting to examine whether autophagy plays a similar role in controlling mitochondrial MEF2D activity. Although MEF2D levels in the whole cell are elevated in the brains of alpha-synuclein transgenic mice and PD patients (29), its levels in the mitochondria are decreased in PD patients. Collectively, these data indicate that the decrease in MEF2D level in mitochondria is accompanied by a buildup of MEF2D in the cytoplasm. It is possible that reduced mitochondrial MEF2D may contribute to the overall increase of cytoplasmic MEF2D. But this effect should be small, since only a small fraction of MEF2D will normally go to mitochondria. Whether and how alpha-synuclein may affect mitochondrial MEF2D requires further investigation.

Our data suggest that MEF2D exclusively regulates expression of the ND6 gene without significant effects on several other proteinencoding mtDNA genes tested. The reason for this high degree of specification is not clear but quite intriguing. Since ND6 is the only protein-encoding gene present in the L strand of $\mathrm{mtDNA}$, whereas the rest of the 13 protein-encoding mitochondrial genes all reside in the $\mathrm{H}$ strand (7), this apparent specificity may be due, at least in part, to the unique organization of mtDNA. Indeed, cAMP 
A

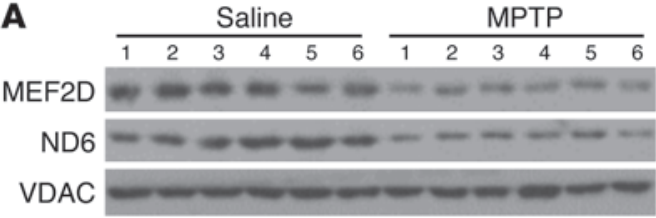

Day 5

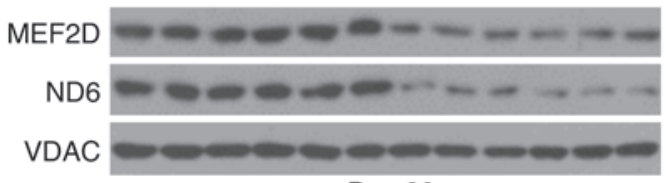

Day 20
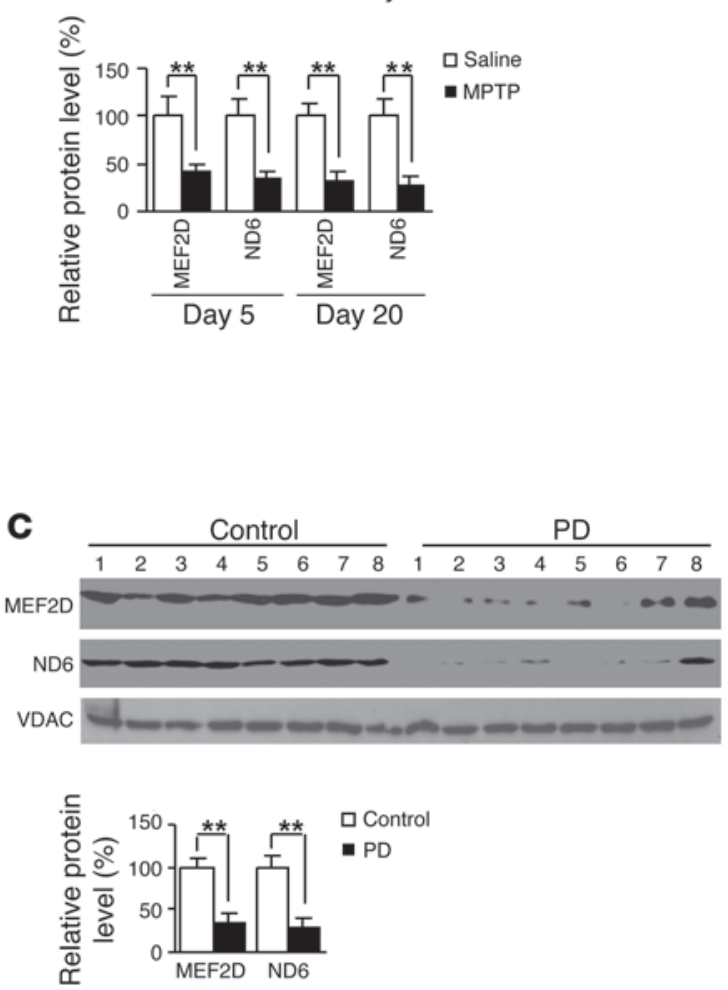

Figure 7

Correlation of mitochondrial MEF2D in a MPTP model of PD and in postmortem brains of PD patients. (A) Reduced mitochondrial MEF2D and ND6 levels in the brains of MPTP-treated mice $\left(n=18 ;{ }^{* \star} P<0.01\right)$. Mitochondria purified from brain $\mathrm{SNpc}$ region were analyzed by Western blotting. Experiments were repeated 3 times. (B) Role of mitochondrial MEF2D-ND6 pathway in maintaining $\mathrm{TH}^{+}$neurons in SNpc in a MPTP mouse model of PD. For each group, 3 mice received stereotactic injection of control vector (GFP) or Mt2Ddn lentivirus in SN. 2 weeks later, mice were exposed to MPTP. After treatment for 7 days, survival of lentivirus-transduced $\mathrm{TH}^{+}$neurons in $\mathrm{SN}$ was determined by immunohistochemistry. Scale bars: $30 \mathrm{um}$. Quantitative analysis of 9 mice from 3 independent experiments is also shown $\left({ }^{\star *} P<0.01\right)$. (C) Reduced mitochondrial MEF2D and ND6 levels in the brains of human PD patients. Mitochondria were purified from brain striata of postmortem PD patients and normal controls. Equal amounts of mitochondrial proteins were subjected to Western blotting. Quantitative analysis of the bands is also shown $(n=13$ patients and 13 controls; ${ }^{*} P<0.01$ ). Experiments were repeated 2 times.
B

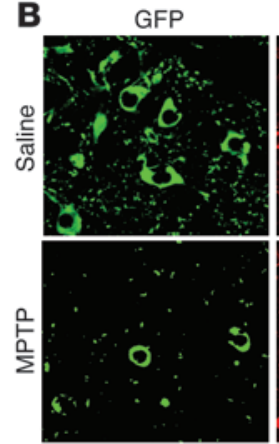

Mt2Ddn

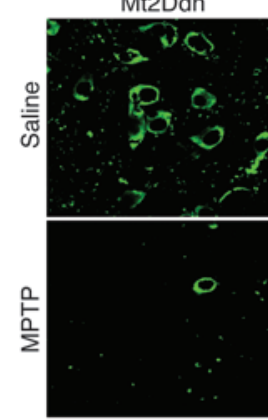

Mt2DVP16
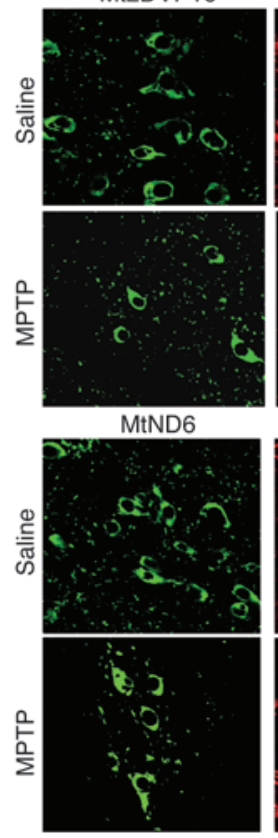

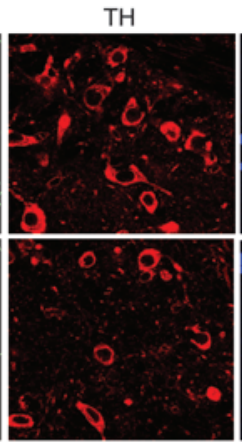

TH

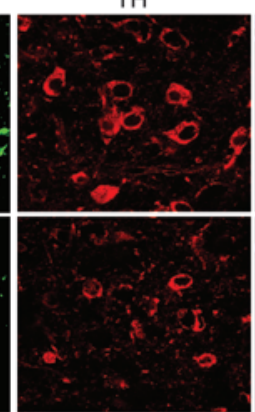

$\mathrm{TH}$
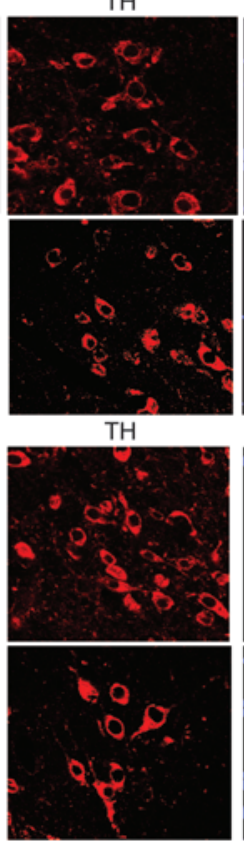

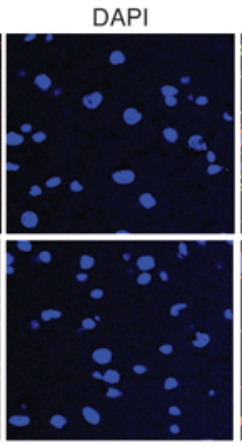

DAPI

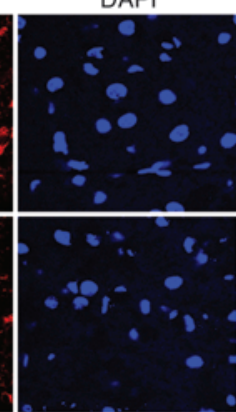

DAPI

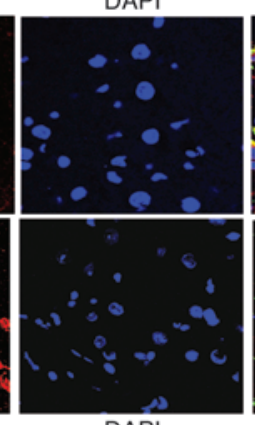

DAPI

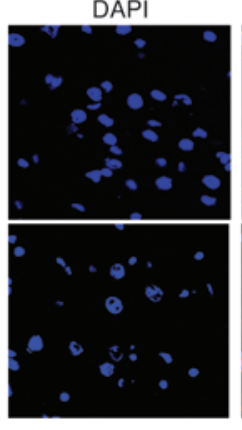

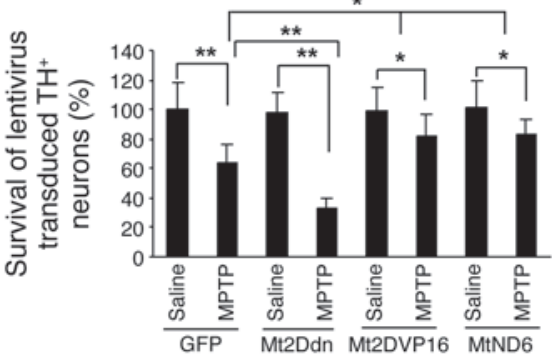
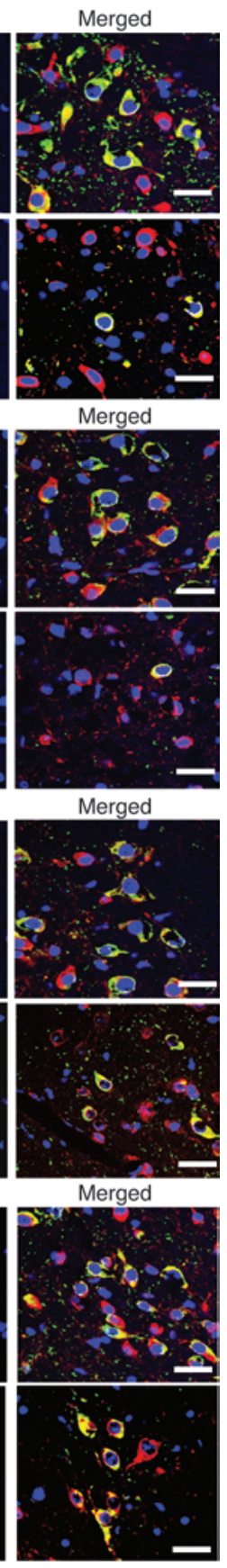
response element-binding protein (CREB) has previously been shown to bind the D-loop, whereas p53 can also localize to mitochondria under stressful conditions. But none of them has been reported to affect L-strand transcription (30-32). Insufficiency of ND6 protein is known to lead to severe disruption of complex I structure (6). Therefore, maintaining adequate levels of ND6 is critical for the proper assembly of complex I (33). Our data clearly showed that a reduction of MEF2D activity specifically in mitochondria resulted in substantial disorganization of complex I and subsequent loss of complex I activity without affecting other complexes. These findings highlight the critical and distinctive role of MEF2D in maintaining the function of complex I. Together with the reported mitochondrial localization by CREB and p53 $(30,31)$, these studies emphasize a previously underappreciated mechanism of interaction between nucleus and mitochondria. The selective degeneration of DA midbrain neurons in the substantia nigra (SN) is a hallmark of PD. DA neurons in the neighboring ventral tegmental area (VTA) are markedly less affected. The mechanisms for this differential vulnerability of DA neurons are unknown. Recent studies have identified several differences between them, including different transcriptional response to MPTP (34), divergent electrophysiological features (35), and selective activation of ATP-sensitive potassium channels (36). It is therefore possible that $\mathrm{SN}$ and VTA DA neurons may have different sensitivity to mitochondrial malfunction induced by MEF2D defects.

Although the full biological significance of this added layer of regulation of $N D 6$ gene expression by MEF2D requires further exploration, our studies offer multiple lines of evidence, including a cellular model, animal studies, and examination of human tissues, to implicate this regulatory mechanism in the pathogenic process of $\mathrm{PD}$. Our results indicated that well-established toxins known to target complex I and induce parkinsonism in model systems also reduced MEF2D levels in mitochondria and disrupted MEF2D binding to the ND6 MEF2 site, offering an alternative mode of action by which these important toxins inhibit mitochondrial function. Because low-dose toxins preferentially reduce mitochondrial MEF2D without affecting its level in the nucleus, our data suggest that inhibition of the MEF2D-ND6 pathway could represent one of the earlier steps involved in pathologic changes at the subcellular level (37). More importantly, the levels of both MEF2D and ND6 proteins were greatly reduced in brain mitochondria of both chronic MPTP-treated mice and human PD patients. These findings are consistent with the notion that reduced complex I activity secondary to toxic agent-induced inhibition of the MEF2D-ND6 pathway may contribute to the mitochondrial dysfunction and oxidative stress often observed in PD and possibly in other neurodegenerative diseases $(3,26)$. The molecular mechanisms by which complex I inhibitors disrupt MEF2D mitochondrial function are presently unclear. Since both MPP+ and rotenone directly bind complex I (38), it is possible that these toxins may exert their effects on MEF2D via a mechanism involving complex I inhibition or disruption of mitochondrial redox balance.

A recent study found that mice with the Mef $2 d$ gene conditionally deleted are viable and show no obvious phenotypic abnormalities (39). However, under stress, these mice showed defects in cardiac remodeling. This is consistent with our data demonstrating that reduced MEF2D activity in mitochondria sensitized the cells to toxic stress. While our data have focused on the regulation of mitochondria by MEF2D in mediating PD-like injury, it is likely that mitochondrial MEF2D plays a role in other organ systems and disease processes as well.

\section{Methods}

Purification of mitochondria and mitochondrial subfractionation. Mitochondria were purified from brain tissue using the discontinuous sucrose gradient method. Briefly, brain homogenate was made in ice-cold homo-buffer $(0.32 \mathrm{M}$ sucrose, $20 \mathrm{mM}$ Tris-HCl, $\mathrm{pH} 7.4$ ) and spun at $900 \mathrm{~g}, 4^{\circ} \mathrm{C}$, for 10 minutes. The supernatant was transferred to another clean tube and spun at $10,000 \mathrm{~g}$, $4^{\circ} \mathrm{C}$, for 10 minutes. The resultant pellet, enriched for mitochondria, was resuspended in $2 \mathrm{ml}$ homo-buffer, loaded on top of a sucrose gradient $(1.2 \mathrm{M}$, $0.8 \mathrm{M}$, and $0.32 \mathrm{M}$ sucrose; $20 \mathrm{mM}$ Tris- $\mathrm{HCl}, \mathrm{pH} 7.4)$ and spun at $53,000 \mathrm{~g}$, $4^{\circ} \mathrm{C}$, for 2 hours. The white band at the interface between medium $(0.8 \mathrm{M})$ and heavy $(1.2 \mathrm{M})$ solutions was collected as highly purified mitochondria. Mitochondria from cultured cells were isolated using a kit (catalog no. 89874) from Pierce. Mitochondrial subfractionation was carried out as described by Hovius et al. (40). Briefly, purified mitochondria (1 mg) were resuspended in $500 \mu \mathrm{l}$ ice-cold buffer $\left(10 \mu \mathrm{M} \mathrm{KH}_{2} \mathrm{PO}_{4}, \mathrm{pH}\right.$ 7.4) and allowed to swell on ice for 20 minutes. Then, 1 volume of iso-osmotic solution ( $32 \%$ sucrose, $30 \%$ glycerol, and $10 \mathrm{mM} \mathrm{MgCl}_{2}$ ) was added, and the mix was spun at $10,000 \mathrm{~g}, 4^{\circ} \mathrm{C}$, for 10 minutes. The supernatant (S1) contained outer membrane and intermembrane space. The pellet (P1) was mitoplasts (matrix surrounded by intact inner membrane). P1 was resuspended in $500 \mu \mathrm{l}$ ice-cold buffer (10 $\left.\mu \mathrm{M} \mathrm{KH}_{2} \mathrm{PO}_{4}, \mathrm{pH} 7.4\right)$ and allowed to swell on ice for 20 minutes. Then, 1 volume of iso-osmotic solution ( $32 \%$ sucrose, $30 \%$ glycerol, and $10 \mathrm{mM} \mathrm{MgCl}_{2}$ ) was added. S1 and the resuspended P1 were spun at $15,000 \mathrm{~g}, 4^{\circ} \mathrm{C}$, for 1 hour. The supernatant from S1 contained the intermembrane space, and the pellet was the outer membrane. The supernatant from P1 contained the matrix, and the pellet was the inner membrane.

Immunofluorescence and immunogold electron microscope. SN4741 cells or primary midbrain DA neurons were plated on glass slides in 24-well plates. Localization of endogenous and exogenous MEF2D was visualized on a Zeiss LSM5 PASCAL confocal microscope. To visualize colocalization of MEF2D with the mitochondria, cells were stained with MitoTracker (Invitrogen) and anti-MEF2D (BD Bioscience) or anti-FLAG (Sigma-Aldrich) antibodies. Quantification of colocalization of signals was done using the "Colocalization Analysis" program of software Image Pro (Media Cybernetics). Overlap coefficients of 50 cells in each group were collected. The overlap coefficient of the control group was set as $100 \%$. For immunogold labeling of MEF2D, rat brains were fixed in $2 \%$ paraformaldehyde and $0.5 \%$ glutaraldehyde, embedded in London Resin White, and sectioned at $80 \mathrm{~nm}$. Sections were incubated in 1:500 dilution of the monoclonal antibody to MEF2D. Goat anti-mouse IgG antibody conjugated to a 5-nm colloidal gold particle (Polysciences) was used as secondary antibody and photographed using a LEO EM-910 transmission electron microscope (LEO Electron Microscopy Inc.) at $80 \mathrm{kV}$. For TEM study on cultured cells, we diluted the anti-MEF2D antibody to 1:500 and goat anti-mouse IgG antibody conjugated to a $15 \mathrm{~nm}$ gold particle (Polysciences) was used as secondary antibody.

In vitro import assay. Mouse MEF2D cDNA was amplified by PCR using primers flanking the coding region. The $\mathrm{T} 7$ phage promoter was incorporated in the sense primer for in vitro transcription of the PCR fragment. In vitro transcription and translation were performed using the TNT T7 Quick Coupled Transcription/Translation System (Promega) in the presence of $20 \mu \mathrm{Ci}\left[{ }^{35} \mathrm{~S}\right]$ methionine (Amersham). Fresh mitochondria prepared from $2 \times 10^{7} \mathrm{SN} 4741$ cells (41) were suspended in incubation buffer (42) to a final protein concentration of $2 \mathrm{mg} / \mathrm{ml}$. The in vitro import assay was then carried out as described previously (42). Samples were separated by SDS-PAGE. After fixation in 10\% acetic acid and 25\% isopropanol, the gel was dried and exposed to HyBlot film (Denville) under $-80^{\circ} \mathrm{C}$ conditions. Mouse mtHsp70 siRNA, MEF2D siRNA, and ChIP assay. Mouse mtHsp70 siRNA was performed using a kit (catalog no. sc-35521) from Santa Cruz following procedures provided by the manufacturer. MEF2D siRNA expressing lentivirus was constructed as described by Tian et al. (43). The 
siRNA against MEF2D targeted the sequence 5'-GTAGCTCTCTGGTCACTCC- $3^{\prime}$. To produce a nonsilencing scrambled siRNA, the sequence of the MEF2D siRNA was altered such that it targeted the sequence 5'-GTATCTCTCTGGTCGCTCC-3' (mismatches in bold) (44). ChIP assay was done using a ChIP Assay Kit (catalog no. 17-295) from Upstate following procedures provided by the manufacturer.

Quantitative real-time PCR. Complementary DNA was generated from total RNA using random priming and MMLV reverse transcriptase (Invitrogen). Quantitative real-time PCR was performed using SYBR Green PCR Master Mix (Invitrogen) and analyzed on an AB Prism 7000 analyzer (Applied Biosystems). All real-time values were normalized to $18 \mathrm{~S}$ rRNA. The real-time oligonucleotides used in the study were: $18 \mathrm{~S}$ forward, 5'-CGGCTACCACATCCAAGGAA-3', 18S reverse, 5'-GAGCTGGAATTACCGCGGCT-3'; ND6 forward, 5'-ATTAAACAACCAACAAACCCAC-3', ND6 reverse, 5'-TTTGGTTGGTTGTCTTGGGTT-3'; ND2 forward, 5'-CCCATTCCACTTCTGATTACC-3', ND2 reverse, 5'-ATAGTAGAGTTGAGTAGCGGG-3'; ATP6 forward, 5'-CATTCTTTCCAACACTGACTAG-3', ATP6 reverse, 5'-GCCTAGGAGATTTGTTGATCC-3'; COX2 forward, 5'-TAGCTCCTTAGTCCTCTATATC-3', COX2 reverse, 5'-TGATAAGGATTACAGCTGGTAG-3'; CYTB forward, 5'-GTCCCTTCTAGGAGTCTGCC-3', CYTB reverse, 5'-TCCGTTTGCGTGTATATATCGG-3'.

In vitro transcription. A linear DNA fragment encompassing $14,560 \mathrm{nt}$ to $477 \mathrm{nt}$ of human mtDNA was used as template for in vitro transcription. Individual reaction mixtures $(25 \mu \mathrm{l})$ contained $2.5 \mathrm{pmol}$ human TFAM, $500 \mathrm{fmol}$ human mtDNA-directed RNA polymerase (POLRMT), and $500 \mathrm{fmol}$ human TFB2M (Enzymax) as well as 1 pmol purified Mt2D or $\mathrm{Mt} 2 \mathrm{Ddn}$ protein. In addition, the reactions contained $10 \mathrm{mM}$ Tris- $\mathrm{HCl}$ ( $\mathrm{pH}$ 8.0), $10 \mathrm{mM} \mathrm{MgCl}, 1 \mathrm{mM}$ DTT, $100 \mu \mathrm{g} / \mathrm{ml}$ bovine serum albumin, 4 U RNA Guard (Roche), $0.4 \mathrm{mM}$ ATP, $0.15 \mathrm{mM}$ CTP, $0.15 \mathrm{mM} \mathrm{GTP}$, $0.01 \mathrm{mM}$ UTP, $1.25 \mu \mathrm{Ci}\left[\alpha^{-32} \mathrm{P}\right]$-UTP, and $85 \mathrm{fmol}$ DNA template (45). After incubation at $32^{\circ} \mathrm{C}$ for 30 minutes, the reactions were stopped by adding $200 \mu \mathrm{l}$ stop buffer (10 mM Tris-HCl, pH 8.0; 0.2 M NaCl; 1 mM EDTA; and $0.1 \mathrm{mg} / \mathrm{ml}$ glycogen). Samples were then incubated with $100 \mu \mathrm{g} / \mathrm{ml}$ proteinase $\mathrm{K}$ in the presence of $0.5 \% \mathrm{SDS}$ at $42^{\circ} \mathrm{C}$ for 45 minutes, and the labeled transcripts were precipitated with $0.6 \mathrm{ml}$ ice-cold ethanol. Pellets were resuspended in $10 \mu \mathrm{l}$ loading buffer $(98 \%$ formamide; $10 \mathrm{mM}$ EDTA, pH 8.0; 0.025\% xylene cyanol; and 0.025\% bromophenol blue), heated at $95^{\circ} \mathrm{C}$ for 4 minutes, kept on ice for 3 minutes, and loaded on a $4 \%$ polyacrylamide $/ 7 \mathrm{M}$ urea gel in $1 \times$ tris-borate-ethylenediaminetetraacetic acid (TBE). After electrophoresis, the gels were dried and exposed to HyBlot film (Denville) under $-80^{\circ} \mathrm{C}$.

In organello transcription assay. SN4741 cells were infected with Mt2D or Mt2Ddn lentiviruses for 24 hour. De novo transcription was measured in isolated mitochondria as described previously $(46,47)$. The mitochondrial fraction was suspended in transcription buffer containing $25 \mathrm{mM}$ sucrose, $75 \mathrm{mM}$ sorbitol, $100 \mathrm{mM} \mathrm{KCl}, 10 \mathrm{mM} \mathrm{K}_{2} \mathrm{HPO}_{4}, 50 \mathrm{mM}$ EDTA, $5 \mathrm{mM} \mathrm{MgCl}_{2}, 1 \mathrm{mM}$ ADP, $10 \mathrm{mM}$ glutamate, $2.5 \mathrm{mM}$ malate, and $10 \mathrm{mM}$ Tris- $\mathrm{HCl}$ ( $\mathrm{pH}$ 7.4), with $1 \mathrm{mg}$ of BSA per milliliter. Mitochondria containing a total $200 \mu \mathrm{g}$ protein were incubated in $300 \mu \mathrm{l}$ of the transcription buffer containing $0.1 \mathrm{mM}$ Bio-11-UTP (Ambion) at $37^{\circ} \mathrm{C}$ for 30 minutes. After incubation, the mitochondria were pelleted; washed with
PBS; solubilized in $100 \mathrm{ml}$ of lysis buffer containing $50 \mathrm{mM}$ Tris- $\mathrm{HCl}$ ( $\mathrm{pH}$ 8.0), $20 \mathrm{mM} \mathrm{NaCl}, 1 \mathrm{mM}$ EDTA, $1 \%$ SDS, and $20 \mathrm{mg}$ of protease $\mathrm{K}$ (Gibco, Invitrogen); and then incubated at room temperature for $15 \mathrm{~min}$ utes. The mitochondrial RNA was isolated by phenol extraction. Biotinylated mitochondrial RNA was isolated by streptavidin (Invitrogen) purification. Reverse transcription and ND6 real-time PCR were done as described above. The primers for detection 16S RNA were 5'-GTACCGCAAGGGAAAGATGAAAG-3' (forward) and 5'-GGTAACCAGCTATCACCAAGCTC-3' (reverse).

MPTP mouse model of PD and postmortem human brain samples. The MPTP mouse model of PD was created as described by Bezard et al. (48). All procedures were approved by the IACUC of Emory University. Postmortem human brain samples obtained with proper consent were provided by the Brain Bank, Center for Neurodegenerative Disease, Emory University. Use of the deidentified archived specimens did not require IRB approval. The control and PD cases matched in age (control, $70.4 \pm 5.3$ years; PD, $72.5 \pm 8.1$ years), race (all mixed European descent), sex (control, 6:7 female/male; PD, 7:6 female/male), and postmortem interval (control, $7.8 \pm 3.4$ hour; PD, $8.3 \pm 3.2$ hour). They were not diagnosed with other neurodegenerative diseases, including Alzheimer disease. The IRB of Emory University approved all procedures, and all subjects signed written informed consent.

Cell culture, plasmid and lentivirus preparation, mitochondrial complex activity and functional assays, electrophoretic mobility shift assay, MEF2 luciferase reporter assay, and WST-1 assay. See Supplemental Methods.

Statistics. Data were expressed as mean \pm SEM. Differences between experimental groups were evaluated using 2-tailed Student's $t$ test. A $P$ value less than 0.05 was considered significant.

\section{Acknowledgments}

This work was partially supported by NIH grants ES015317, AG023695, and NS048254 (to Z. Mao) and ES016731 (to G. Miller and Z. Mao), The Robert W. Woodruff Health Sciences Center Fund (to Z. Mao), Michael J. Fox Foundation (to Z. Mao), and Viral Vector, Tissue Bank, and Imaging Cores of the Emory Neuroscience NINDS Core Facilities grant P30NS055077. We thank Pier Giorgio Mastroberardino for help with mitochondria purification, Hoon Ryu for pDsRed2-Mito plasmid, Howard Rees for help with confocal microscopy, James Greene for critical reading of the manuscript, Megan Dautherty for assistance with immunocytochemistry, Yingjie Li and Minzheng Wang for animal studies, and Jean-Francois Pare and Hong Yi for assistance with electron microscopy.

Received for publication May 28, 2010, and accepted in revised form December 15, 2010.

Address correspondence to: Zixu Mao, Department of Pharmacology, Emory University, 615 Michael Street, Atlanta, Georgia 30322, USA. Phone: 404.712.8581; Fax: 404.727.3728; E-mail: zmao@ pharm.emory.edu.
1. Chan DC. Mitochondria: dynamic organelles in disease, aging, and development. Cell. 2006; 125(7):1241-1252.

2. Manfredi G, Beal MF. Merging mitochondria for neuronal survival. Nat Med. 2007;13(10):1140-1141.

3. Schapira AH. Mitochondrial disease. Lancet. 2006;368(9529):70-82.

4. Orrenius S, Gogvadze V, Zhivotovsky B. Mitochondrial oxidative stress: implications for cell death. Annu Rev Pharmacol Toxicol. 2007;47:143-183.

5. Mattson MP, Gleichmann M, Cheng A. Mitochon- dria in neuroplasticity and neurological disorders. Neuron. 2008;60(5):748-766.

6. Bai Y, Attardi G. The mtDNA-encoded ND6 subunit of mitochondrial NADH dehydrogenase is essential for the assembly of the membrane arm and the respiratory function of the enzyme. EMBOJ. 1998;17(16):4848-4858.

7. Falkenberg M, Larsson NG, Gustafsson CM. DNA replication and transcription in mammalian mitochondria. Annu Rev Biochem. 2007;76:679-699.

8. Jun AS, Brown MD, Wallace DC. A mitochondrial
DNA mutation at nucleotide pair 14459 of the NADH dehydrogenase subunit 6 gene associated with maternally inherited Leber hereditary optic neuropathy and dystonia. Proc Natl Acad Sci U S A. 1994;91(13):6206-6210.

9. Kosel S, et al. Novel mutations of mitochondrial complex I in pathologically proven Parkinson disease. Neurogenetics. 1998;1(3):197-204.

10. Keeney PM, Xie J, Capaldi RA, Bennett JP Jr. Parkinson's disease brain mitochondrial complex I has oxidatively damaged subunits and is func- 
tionally impaired and misassembled. J Neurosci. 2006;26(19):5256-5264.

11. Shadel GS. Coupling the mitochondrial transcription machinery to human disease. Trends Genet. 2004;20(10):513-519.

12. Potthoff MJ, Olson EN. MEF2: a central regulator of diverse developmental programs. Development. 2007;134(23):4131-4140

13. Heidenreich KA, Linseman DA. Myocyte enhancer factor-2 transcription factors in neuronal differentiation and survival. Mol Neurobiol. 2004;29(2):155-166.

14. Mao Z, Bonni A, Xia F, Nadal-Vicens M, Greenberg ME. Neuronal activity-dependent cell survival mediated by transcription factor MEF2. Science. 1999;286(5440):785-790.

15. Gong X, et al. Cdk5-mediated inhibition of the protective effects of transcription factor MEF2 in neurotoxicity-induced apoptosis. Neuron. 2003;38(1):33-46.

16. Liu L, Cavanaugh JE, Wang Y, Sakagami H, Mao Z, Xia Z. ERK5 activation of MEF2-mediated gene expression plays a critical role in BDNF-promoted survival of developing but not mature cortical neurons. Proc Natl Acad Sci U S A. 2003;100(14):8532-8537.

17. Wang X, Tang X, Li M, Marshall J, Mao Z. Regulation of neuroprotective activity of myocyteenhancer factor 2 by cAMP-protein kinase A signaling pathway in neuronal survival. $\mathrm{J}$ Biol Chem. 2005;280(17):16705-16713.

18. Tang X, et al. Cyclin-dependent kinase 5 mediates neurotoxin-induced degradation of the transcription factor myocyte enhancer factor 2. J Neurosci. 2005;25(19):4823-4834.

19. Smith PD, et al. Calpain-regulated $\mathrm{p} 35 / \mathrm{cdk} 5$ plays a central role in dopaminergic neuron death through modulation of the transcription factor myocyte enhancer factor 2. J Neurosci. 2006;26(2):440-447.

20. McKinsey TA, Zhang CL, Olson EN. MEF2: a calcium-dependent regulator of cell division, differentiation and death. Trends Biochem Sci. 2002;27(1):40-47.

21. Naya FJ, et al. Mitochondrial deficiency and cardiac sudden death in mice lacking the MEF2A transcription factor. Nat Med. 2002;8(11):1303-1309.

22. Son JH, Chun HS, Joh TH, Cho S, Conti B, Lee JW Neuroprotection and neuronal differentiation studies using substantia nigra dopaminergic cells derived from transgenic mouse embryos. J Neurosi. 1999;19(1):10-20.
23. Neupert W, Herrmann JM. Translocation of proteins into mitochondria. Annu Rev Biochem. 2007; 76:723-749.

24. Matouschek A, Pfanner N, Voos W. Protein unfolding by mitochondria. The Hsp70 import motor. EMBO Rep. 2000;1(5):404-410.

25. Andres V, Cervera M, Mahdavi V. Determination of the consensus binding site for MEF2 expressed in muscle and brain reveals tissue-specific sequence constraints. J Biol Chem. 1995;270(40):23246-23249.

26. Abou-Sleiman PM, Muqit MM, Wood NW. Expanding insights of mitochondrial dysfunction in Parkinson's disease. Nat Rev Neurosci. 2006;7(3):207-219.

27. Liberatore GT, et al. Inducible nitric oxide synthase stimulates dopaminergic neurodegeneration in the MPTP model of Parkinson disease. Nat Med. 1999; 5(12):1403-1409.

28. Betarbet R, Sherer TB, MacKenzie G, Garcia-Osuna M, Panov AV, Greenamyre JT. Chronic systemic pesticide exposure reproduces features of Parkinson's disease. Nat Neurosci. 2000;3(12):1301-1306.

29. Yang $Q$, et al. Regulation of neuronal survival factor MEF2D by chaperone-mediated autophagy. Science. 2009;323(5910):124-127

30. Ryu H, Lee J, Impey S, Ratan RR, Ferrante RJ Antioxidants modulate mitochondrial PKA and increase CREB binding to D-loop DNA of the mitochondrial genome in neurons. Proc Natl Acad Sci U S A. 2005;102(39):13915-13920.

31. Leu JI, Dumont P, Hafey M, Murphy ME, George DL. Mitochondrial p53 activates Bak and causes disruption of a Bak-Mcl1 complex. Nat Cell Biol. 2004;6(5):443-450.

32. Mihara M, et al. p53 has a direct apoptogenic role at the mitochondria. Mol Cell. 2003;11(3):577-590.

33. Kruse SE, Watt WC, Marcinek DJ, Kapur RP, Schenkman KA, Palmiter RD. Mice with mitochondrial complex I deficiency develop a fatal encephalomyopathy. Cell Metab. 2008;7(4):312-320.

34. Phani S, Gonye G, Iacovitti L. VTA neurons show a potentially protective transcriptional response to MPTP. Brain Res. 2010;1343:1-13.

35. Korotkova TM, Ponomarenko AA, Brown RE, Haas HL. Functional diversity of ventral midbrain dopamine and GABAergic neurons. Mol Neurobiol. 2004;29(3):243-259.

36. Liss B, Haeckel O, Wildmann J, Miki T, Seino S,
Roeper J. K-ATP channels promote the differential degeneration of dopaminergic midbrain neurons. Nat Neurosci. 2005;8(12):1742-1751.

37. Yuan J, Yankner BA. Apoptosis in the nervous system. Nature. 2000;407(6805):802-809.

38. Richardson JR, Quan Y, Sherer TB, Greenamyre JT, Miller GW. Paraquat neurotoxicity is distinct from that of MPTP and rotenone. Toxicol Sci. 2005;88(1):193-201.

39. Kim Y, et al. The MEF2D transcription factor mediates stress-dependent cardiac remodeling in mice. J Clin Invest. 2008;118(1):124-132.

40. Hovius R, Lambrechts H, Nicolay K, de Kruijff B. Improved methods to isolate and subfractionate rat liver mitochondria. Lipid composition of the inner and outer membrane. Biochim Biophys Acta. 1990;1021(2):217-226.

41. Fernandez-Vizarra E, Lopez-Perez MJ, Enriquez JA. Isolation of biogenetically competent mitochondria from mammalian tissues and cultured cells. Methods. 2002;26(4):292-297.

42. Petruzzella V, Tiranti V, Fernandez P, Ianna P, Carrozzo R, Zeviani $\mathrm{M}$. Identification and characterization of human cDNAs specific to BCS1, PET112, SCO1, COX15, and COX11, five genes involved in the formation and function of the mitochondrial respiratory chain. Genomics. 1998;54(3):494-504.

43. Tian B, Yang Q, Mao Z. Phosphorylation of ATM by Cdk5 mediates DNA damage signalling and regulates neuronal death. Nat Cell Biol. 2009;11(2):211-218.

44. Flavell SW, et al. Activity-dependent regulation of MEF2 transcription factors suppresses excitatory synapse number. Science. 2006;311(5763):1008-1012.

45. Gaspari M, Falkenberg M, Larsson NG, Gustafsson $\mathrm{CM}$. The mitochondrial RNA polymerase contributes critically to promoter specificity in mammalian cells. EMBO J. 2004;23(23):4606-4614.

46. Park CB, et al. MTERF3 is a negative regulator of mammalian mtDNA transcription. Cell. 2007; 130(2):273-285.

47. Gaines G, Attardi G. Highly efficient RNA-synthesizing system that uses isolated human mitochondria: new initiation events and in vivo-like processing patterns. Mol Cell Biol. 1984;4(8):1605-1617.

48. Bezard E, Dovero S, Bioulac B, Gross CE. Kinetics of nigral degeneration in a chronic model of MPTPtreated mice. Neurosci Lett. 1997;234(1):47-50. 